\title{
Prevalence, natural history, and management of suspected barrett's esophagus in patients with cirrhosis
}

\begin{abstract}
Background and Aims: Barrett's esophagus is a pre-malignant condition with increased risk of esophageal cancer in which metaplastic intestinal mucosa replaces the normal esophageal squamous mucosa. It is suspected during endoscopy when salmon colored mucosa is seen in the esophagus, but requires biopsy for confirmation. In the presence of cirrhosis, biopsies are often limited by coagulopathy, thrombocytopenia, and presence of esophageal varices, which makes the diagnostic and therapeutic options for Barrett's esophagus problematic. The purpose of this study is to look at the prevalence of suspected Barrett's esophagus in patients with cirrhosis, assess how often it was confirmed with biopsy, and evaluate its natural history and progression to adenocarcinoma. There have been no previous similar studies.
\end{abstract}

Methods: Our study involves a retrospective design to collect data of patients with cirrhosis who underwent upper endoscopy (EGD) at Saint Louis University and were found to have Barrett's esophagus, either suspected or confirmed. Data analysis was then completed to compare demographics, risk factors, natural history, and survival of the two groups.

Results: Our data suggests that the prevalence of Barrett's esophagus in patients with liver cirrhosis is increased compared to that found in the general population. Risk factors for the development and confirmation of Barrett's esophagus, along with survival data, were compared between groups and were not found to be statistically significant.

Conclusion: A higher prevalence of Barrett's esophagus is noted within a population of cirrhotics undergoing endoscopy. The estimated prevalence of Barrett's esophagus is $0.076-2 \%$ in the general population undergoing endoscopy whereas our study demonstrated a prevalence of over $10 \%$ of potential Barrett's patients $(1.9 \%$ confirmed). It is important to recognize possible shared risk factors and the increased prevalence of Barrett's esophagus in this patient population.

Keywords: esophageal adenocarcinoma, premalignant condition, esophageal cancer, biopsies and clinical characteristics, intestinal metaplasia, alcohol consumption, data analysis, meld score, coagulopathy, thrombocytopenia, hepatocellular carcinoma, endoscopic surveillance, plasma vasoactive peptides, neurotensin, liver cirrhosis, hiatal hernia
Volume 4 Issue I - 2018

Ruchi Bhatia,' Hisham Hussan, ${ }^{2}$ Robert Hilton, ${ }^{3}$ Jalpan Ringwala, ${ }^{4}$ Justin Yu BS, ${ }^{5}$ Kara M Christopher, ${ }^{6}$ Adrian M Di Bisceglie,' Christine Hachem'

'Department of Internal Medicine, Saint Louis University School of Medicine, USA

${ }^{2}$ Division of Gastroenterology, Hepatology, and Nutrition, Ohio State University Medical Center, USA

${ }^{3}$ Department of Internal Medicine, Robert Wood Johnson University Hospital, USA

${ }^{4}$ Department of Internal Medicine, Boston Medical Center, USA ${ }^{5}$ Department of Internal Medicine, Saint Louis University School of Medicine, USA

${ }^{6}$ Saint Louis University Cancer Center, USA

Correspondence: Christine Hachem, Associate Professor of Medicine, Division of Gastroenterology and Hepatology, Department of Internal Medicine, Saint Louis University School of Medicine, Saint Louis, Missouri, USA, Tel 3|4-606-397।, Fax 3|4-577-8|25, Email christine.hachem@health.slu.edu

Ruchi Bhatia, Division of Gastroenterology and Hepatology, Department of Internal Medicine, 4465 West Pine Blvd Apt 14 St. Louis MO 63108, Saint Louis University School of Medicine, Saint Louis, Missouri, USA, Tel 6146680295,

Email rbhatia714@gmail.com

Received: October 27, 2017 | Published: January 19, 2018
Abbreviations: IM, intestinal metaplasia; EAC, esophageal adenocarcinoma; HGD, high grade dysplasia; LGD, low grade dysplasia; GAVE, gastric astral vascular ectasia; GERD, gastroesophageal acid reflux disease; PPI, proton pump inhibitor; HCC, hepatocellular carcinoma; ASGE, american society of gastrointestinal endoscopy; BE, barrett's esophagus; EGD: Esophagogastroduodenoscopy; NASH, non-alcoholic steatohepatitis

\section{Introduction}

Esophageal cancer is the sixth leading cause of cancer mortality worldwide. ${ }^{1}$ While esophageal squamous cell carcinoma rates are declining, the incidence of esophageal adenocarcinoma has increased by six fold between 1975 to $20011^{2,3}$ Barrett's esophagus (BE) is a premalignant condition that can lead to esophageal adenocarcinoma (EAC) in $0.018 \%$ of patients per year; recognizing and preventing its progression to esophageal adenocarcinoma has been a major focus to tackle increasing rates of esophageal cancer. The estimated prevalence of $\mathrm{BE}$ is $0.076-2 \%$ in the general population undergoing endoscopy for any reason. ${ }^{4}$ Risk factors for the development of BE include long standing gastroesophageal reflux disease (GERD), male gender, obesity, and older age. ${ }^{5-7}$ Many of these risk factors are shared with cirrhotic patients. It is then not surprising that patients with cirrhosis are also at high risk of esophageal cancer, with a 2.6 to 7.5 fold increased incidence of developing esophageal cancer compared to controls. ${ }^{8-10}$ Furthermore, patients with cirrhosis and esophageal cancer have higher chemotherapy related toxicities when compared to patients without cirrhosis. ${ }^{11}$ However, there is limited data in the literature on whether the risk of esophageal cancer in cirrhosis is due to adenocarcinoma or squamous cell carcinoma. In addition, prevalence, risk factors, and natural history of Barrett's esophagus have not been well defined in patients with cirrhosis. This may be due to potential difficulty in the diagnosis and surveillance of $\mathrm{BE}$ in cirrhotic patients due to factors such as esophageal varices, thrombocytopenia or coagulopathy. ${ }^{12}$ Thus, we conducted a 
retrospective, comparative cohort study of patients with cirrhosis who underwent esophagogastroduodenoscopy (EGD) for any indication in order to assess prevalence, management, and progression of $\mathrm{BE}$ in patients with cirrhosis.

\section{Methods}

\section{Study design and data collection}

Approval from the Institutional Review Board was obtained before conduction of this study. For this study we included patients with a history of cirrhosis, regardless of etiology, who underwent upper gastrointestinal endoscopy (EGD) at Saint Louis University from $1 / 1 / 2001$ through $12 / 31 / 2008$. We chose to halt data collection at the year 2008 to allow calculation of a 5 year survival rate after the first EGD that was documented at our institution.

Our cohort consisted of patients between 18-80years old, of both genders and all races. To identify patients with cirrhosis, we used billing codes for cirrhosis and CPT codes for EGDs done for any indication during our inclusion period. In order to validate our cohort, we further searched our endoscopy database for any EGD with indications of or findings suggestive of cirrhosis. Results of liver biopsies and clinical characteristics of all these patients were then collected. Patients were then excluded if they had no documented diagnosis of cirrhosis based on laboratory tests (such as serum albumin less than $3.0 \mathrm{~g} / \mathrm{dL}$ or blood platelet counts less than $150,000 \mathrm{~mm}^{3}$ ), radiologic data, or pathologically by demonstration of cirrhosis on liver biopsy when performed. ${ }^{13,14}$

We further divided our cohort in to two study groups including those with and without Barrett's. Among patients in the cohort described above, we searched our medical records for the earliest date of any suspected but not biopsied BE (SBE), confirmed Barrett's esophagus with intestinal metaplasia (IM), BE with dysplasia or esophageal adenocarcinoma (EAC). We also documented corresponding endoscopic findings on initial and subsequent endoscopies for the next 5years. EGD findings were collected also for cirrhotic patients with no evidence of $\mathrm{BE}$ or its complications, including dysplasia and adenocarcinoma. Finally, results of liver biopsies and clinical characteristics of patients were collected including date of liver transplantation if applicable.

\section{Definitions}

Barrett's esophagus (BE) was diagnosed when islands, tongues, or circular extension of salmon colored epithelium was seen above the gastroesophageal junction on EGD along with the presence of intestinal metaplasia with goblet cells on esophageal biopsies. ${ }^{15}$ Suspected but not biopsied BE (SBE) was defined as the presence of characteristic endoscopic findings as above without confirmatory biopsy for BE. We subdivided BE and SBE into long segment when it extended $\geq 3 \mathrm{~cm}$ above the GE junction and short segment $(<3 \mathrm{~cm})$.

\section{Study variables}

Pathology findings that were recorded included the presence of intestinal metaplasia (IM), low grade dysplasia (LGD), high grade dysplasia (HGD), esophageal adenocarcinoma (EAC) with grade and stage, liver biopsy if available (grade, stage and etiology if available). We also documented corresponding endoscopic findings on initial and subsequent endoscopies for the next 5years. EGD findings included date of EGD, indication, presence of salmon colored mucosa, esophagitis, gastric astral vascular ectasia (GAVE), portal hypertensive gastropathy (mild, moderate, severe), hiatal hernia, esophageal and/or gastric varices.

Clinical information collected included age, gender, race, height, weight, smoking status (defined as never, past or current), alcohol consumption (never, social, previously heavy or currently heavy), presence of heartburn or gastroesophageal acid reflux disease (GERD), proton pump inhibitor (PPI) use, aspirin use, etiology of cirrhosis, presence of ascites, presence of hepatocellular carcinoma (HCC), laboratory data in the 1year before and after EGD, liver transplant status and mortality.

\section{Outcomes}

The primary outcome evaluated in this study was the prevalence of suspected $\mathrm{BE}$ and biopsy proven $\mathrm{BE}$ in patients with cirrhosis undergoing EGD for any indication. Our secondary outcomes were to look at potential risk factors for the development of BE in cirrhosis, factors which might have influenced the decision of endoscopist not to confirm BE by biopsy or surveying BE as well as 5year survival rates of cirrhotic patients with and without BE.

\section{Data analysis}

Patient characteristics were summarized using frequencies (percents) for categorical data and means (standard deviation) for continuous variables. Data analysis was completed using SPSS version 23 (IBM Corp, 2015). Statistical significance was defined as alpha $<.05$. Chi-square tests for categorical variables and independent samples t-tests or one-way analysis of variance (ANOVA) for continuous variables were used to examine differences between patients with and without BE, as well as differences between patients with suspected vs. confirmed BE. Cases with missing values on relevant variables were excluded from analysis. Kaplan-Meier survival analysis was conducted to examine differences in survival among those with BE compared to those without BE. Data were rightcensored at last follow up date, which ranged from 0 to 180 months from EGD date (Figure 1).

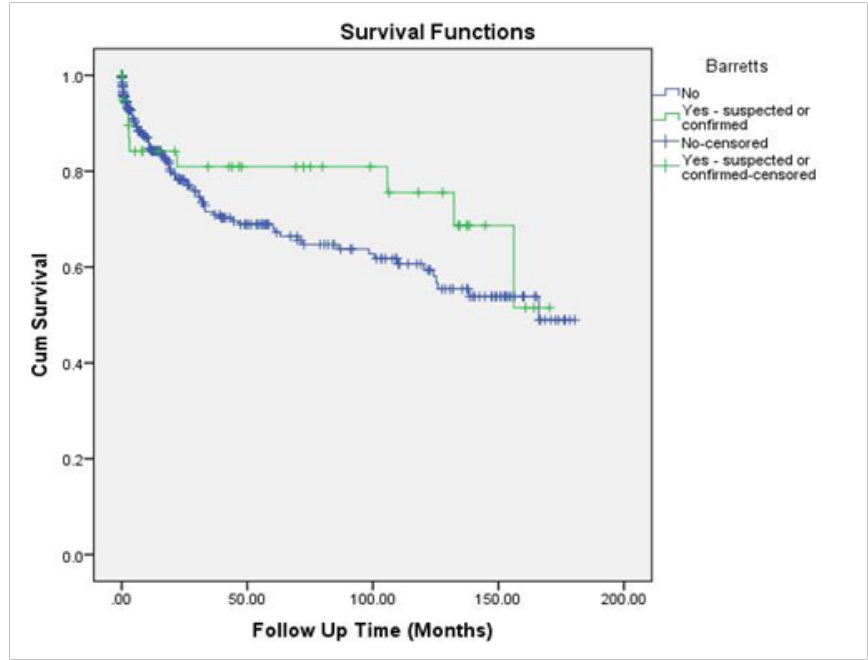

Figure I Survival Functions.

Kaplan-Meier survival analysis was conducted to examine differences in survival among those with BE compared to those without BE. Data were right-censored at last follow up date, which ranged from 0 to 180 months from EGD date. 


\section{Results}

There were a total of 885 patients in our cohort, $76(8.6 \%)$ of which had suspected BE and 17(1.9\%) with confirmed BE. Four patients out of $885(0.004 \%)$ were found to have evidence of esophageal adenocarcinoma on EGD. Of the 17 patients with biopsied BE, $16(94.1 \%)$ were noted to have no dysplasia, 1(5.9\%) with LGD, and none had HGD. None of our patients underwent ablation for the treatment of BE.

Basic demographics, specifically age, gender, and race, were similar between groups as shown in Table 1. The patients had diverse etiologies of cirrhosis with the most common being hepatitis $\mathrm{C}$ (51.3\%), alcohol (18.7\%), and non-alcoholic steatohepatitis (NASH) $(8.8 \%)$ in all groups.

Table I Prevalence of suspected and confirmed Barrett's Esophagus versus no Barrett's

\begin{tabular}{llll}
\hline & Barrett's esophagus & No barrett's & p-value \\
\hline $\begin{array}{l}\text { Mean Age (SD)* } \\
\text { Gender, n(\%) }\end{array}$ & $53.14(10.94)$ & $53.73(10.63)$ & 0.61 \\
$\begin{array}{l}\text { Male } \\
\text { Female }\end{array}$ & $55(59.1)$ & & \\
Race, n(\%) & $34(36.6)$ & $446(56.3)$ & 0.86 \\
White & $49(52.7)$ & $287(36.2)$ & \\
Black & $21(22.6)$ & $423(53.4)$ & \\
Other & $11(11.8)$ & $193(24.4)$ & 0.97 \\
\hline
\end{tabular}

*Missing age for 5 patients
Risk factors for the development of BE were compared between groups, shown in Table 2. In our cohort of patients with confirmed or suspected BE, 4(4.3\%) patients reported social alcohol use, 9(9.6\%) previous heavy use, and $10(10.7 \%)$ current heavy alcohol use. Although not statistically significant, BMI was numerically higher in patients with confirmed BE (mean 34.78) vs suspected BE (27.05) or no BE (29.17).

A MELD score of 12 was chosen as the score where patients began to progress to an advanced degree of cirrhosis with some evidence of clinical decompensation. In our study population, patients with a MELD score greater than 12 as opposed to a lower MELD score were more likely to have suspected $\mathrm{BE}$ than both confirmed $\mathrm{BE}$ or no $\mathrm{BE}$.

We reviewed factors that might have prevented confirming $\mathrm{BE}$ with biopsies and found they were similar between suspected and confirmed BE groups (Table 3). These included coagulopathy, thrombocytopenia, presence of esophageal varices and presence of hepatocellular carcinoma, elevated MELD score, and transplant status. In our cohort of patients with BE (confirmed or suspected), 58 patients $(62.3 \%)$ had esophageal varices, 48 patients $(51.6 \%)$ of which were classified as small esophageal varices and $10(10.7 \%)$ as large varices. In addition, eight $(8.7 \%)$ patients with suspected or confirmed BE had gastric varices. Coagulopathy, defined as an INR greater than 1.5, was present in $14(35.0 \%)$ patients with suspected $\mathrm{BE}$ and $4(30.8 \%)$ patients with confirmed BE.

In order to assess differences in survival in those with confirmed or suspected BE compared to those without BE, a Kaplan-Meier survival analysis was conducted. There was no significant difference in the groups using the log-rank test $(\mathrm{p}=0.30$,). Five year survival was $80.9 \%$ in the BE group compared to $68.9 \%$ in those without BE.

Table 2 Potential factors associated with Barrett's Esophagus

\begin{tabular}{|c|c|c|c|c|}
\hline \multicolumn{5}{|c|}{ Barrett's esophagus } \\
\hline & Suspected $(n=76)$ & Confirmed $(n=18)$ & No Barrett's(n=794) & p-value \\
\hline Mean Age (SD)* & $53.00(11.72)$ & $53.76(6.57)$ & $53.73(10.63)$ & 0.85 \\
\hline \multicolumn{5}{|l|}{ Gender } \\
\hline Male & $49(9.8)$ & $6(1.2)$ & $446(89.0)$ & \multirow{2}{*}{0.16} \\
\hline Female & $25(7.8)$ & $9(2.8)$ & $287(89.4)$ & \\
\hline BMI, mean $(\mathrm{SD}) *$ & $27.05(7.36)$ & $34.78(7.95)$ & $29.17(7.76)$ & 0.15 \\
\hline \multicolumn{5}{|l|}{ Cirrhosis cause } \\
\hline Alcohol & $10(7.5)$ & $I(0.7)$ & $123(91.8)$ & \\
\hline $\mathrm{NASH}$ & $7(11.1)$ & $2(3.2)$ & $54(85.7)$ & \\
\hline Hepatitis C & $28(7.6)$ & $8(2.2)$ & $331(90.2)$ & \\
\hline \multicolumn{5}{|c|}{ GERD/Heartburn, n(\%) } \\
\hline No & $21(2.4)$ & $4(0.45)$ & $272(30.7)$ & \multirow{2}{*}{0.73} \\
\hline Yes & $10(1.1)$ & $2(0.23)$ & $98(I I .1)$ & \\
\hline \multicolumn{5}{|l|}{ Smoking, n(\%) } \\
\hline Never Smoker & $\mathrm{II}(7.6)$ & $4(2.8)$ & $129(89.6)$ & \multirow{3}{*}{0.44} \\
\hline Past Smoker & $10(7.9)$ & $\mathrm{I}(0.8)$ & $113(91.1)$ & \\
\hline Current Smoker & $5(4.4)$ & $\mathrm{I}(0.9)$ & $108(94.7)$ & \\
\hline \multicolumn{5}{|l|}{ PPI, n (\%) } \\
\hline No & $15(7.5)$ & $4(2.0)$ & $182(90.5)$ & \multirow{2}{*}{0.99} \\
\hline Yes & $16(7.4)$ & $4(1.9)$ & $196(90.3)$ & \\
\hline
\end{tabular}


Table Continued....

\begin{tabular}{|c|c|c|c|c|}
\hline \multicolumn{5}{|l|}{ Barrett's esophagus } \\
\hline & Suspected $(n=76)$ & Confirmed $(n=\mid 8)$ & No Barrett's(n=794) & p-value \\
\hline \multicolumn{5}{|l|}{ Aspirin use, n (\%) } \\
\hline No & $24(7.2)$ & $6(1.8)$ & $302(91.0)$ & \multirow{2}{*}{0.94} \\
\hline Yes & $6(8.0)$ & $\mathrm{I}(\mathrm{I} .3)$ & $68(90.7)$ & \\
\hline \multicolumn{5}{|l|}{ Hiatal Hernia, n (\%) } \\
\hline No & $63(8.1)$ & $16(2.1)$ & $700(89.9)$ & \multirow{2}{*}{0.32} \\
\hline Yes & $12(12.1)$ & I (I.0) & $86(86.9)$ & \\
\hline \multicolumn{5}{|l|}{ MELD Score } \\
\hline Less than or equal to 12 & $9(4.8)$ & $4(2.1)$ & $176(93.1)$ & \multirow{2}{*}{0.1} \\
\hline Greater than 12 & $29(10.3)$ & $6(2.1)$ & $247(87.6)$ & \\
\hline
\end{tabular}

*Missing age for 5 patients, BMI missing for 710 patients

Table 3 Potential factors contributing to unconfirmed Barrett's esophagus

\begin{tabular}{|c|c|c|c|}
\hline \multicolumn{4}{|l|}{ Barrett's esophagus } \\
\hline & Suspected $(n=76)$ & Confirmed(n=I7) & p-value \\
\hline \multicolumn{4}{|l|}{ INR, n (\%) } \\
\hline$<1.5$ & $26(34.2)$ & $9(52.9)$ & \multirow{2}{*}{0.78} \\
\hline$>1.5$ & $14(\mid 8.4)$ & $4(23.5)$ & \\
\hline \multicolumn{4}{|l|}{ Platelets, n (\%) } \\
\hline$<50$ & $6(7.9)$ & $0(0)$ & \multirow{2}{*}{0.15} \\
\hline$>50$ & $37(48.6)$ & $13(76.4)$ & \\
\hline \multicolumn{4}{|c|}{ Esophageal Varices, n (\%) } \\
\hline None & $29(38.2)$ & $6(35.3)$ & \multirow{2}{*}{0.83} \\
\hline Present & $47(61.8)$ & II(64.7) & \\
\hline \multicolumn{4}{|l|}{ HCC, n (\%) } \\
\hline No & $32(42.1)$ & $8(47.1)$ & \multirow{2}{*}{0.53} \\
\hline Yes & $8(10.5)$ & $\mathrm{I}(5.8)$ & \\
\hline MELD Score, mean (SD)* & I8.05(8.09) & $13.60(6.40)$ & 0.11 \\
\hline \multicolumn{4}{|l|}{ Transplant, n (\%) } \\
\hline No & $35(46.0)$ & $10(58.8)$ & \multirow{2}{*}{0.46} \\
\hline Yes & $13(17.1)$ & $2(11.8)$ & \\
\hline
\end{tabular}

*Missing data on 45 patients

\section{Discussion}

Our study is the first to look at prevalence of BE and SBE in cirrhotic patients with longitudinal data. Our data suggests that the prevalence of BE in patients with liver cirrhosis is increased compared to that found in the general population. Historically, the estimated prevalence of Barrett's esophagus is $0.076-2 \%$ in the general population undergoing endoscopy whereas our study demonstrated a prevalence of over $10 \%$ of potential Barrett's in cirrhotic patients (1.9\% confirmed). The mechanism of increased prevalence of $\mathrm{BE}$ in patients with liver cirrhosis has not been fully elucidated. As our results show, BE and EAC may have a higher prevalence in cirrhotic patients compared to the general population due to shared risk factors (gender, race, BMI). This may also be due in part to the development of esophageal motility disorders and decreased lower esophageal sphincter pressure seen with varices leading to decreased esophageal clearance and increased contact time between acid and mucosa as well as the increased intra-abdominal pressure from ascites contributing to increase GERD. ${ }^{15-17}$ Some studies have also shown the levels of plasma vasoactive peptides and neurotensin, which are known to decrease the pressure of the lower esophageal sphincter, were markedly higher in patients with cirrhosis than in the normal population. ${ }^{18}$ This likely leads to increased acid exposure and increased development of BE. In addition, the number of cirrhotic patients undergoing routine endoscopy is higher than the general population due to recommendations for variceal screening and surveillance per American College of Gastroenterology (ACG) and American Society of Gastrointestinal Endoscopy (ASGE) guidelines. ${ }^{19,20}$ This may also have contributed in part to the elevated prevalence of BE and EAC. Given the increased prevalence, endoscopic surveillance following 
transplant in cirrhotic patients undergoing liver transplantation should be considered.

BE was confirmed by biopsy in only $1.9 \%$ of our patients. We suspect this to be due to certain risk factors limiting confirmatory biopsy, including the presence of esophageal varices, thrombocytopenia, and coagulopathy. We did not find a statistical association between these factors and the performance of a biopsy for confirmation of BE. The concern of the endoscopist to biopsy a patient with underlying cirrhosis, regardless of varices or lab abnormalities, appears to have played a role in avoiding biopsy. As a result of these factors, cirrhotic patients may not be getting adequate surveillance of BE. The ACG guidelines suggest those patients with chronic GERD and two or more risk factors for $\mathrm{BE}$ or EAC: age $>50$, central obesity, family history, tobacco, etc qualify for screening for Barrett's. ${ }^{19}$ As shown, patients with liver dysfunction have higher risk of progression of $\mathrm{BE}$ and may in fact need more frequent surveillance. Further prospective studies are needed to confirm this association.

As this is a retrospective study, it has several limitations. Potential limitations of our study include a retrospective single institution chart review, along with limited number of patients with biopsy confirmed $\mathrm{BE}$ which in turn limited our evaluation of potential BE progression in a cirrhotic cohort. We do not have information regarding procedural complications. In addition, we initially identified patients with cirrhosis by billing codes. However, we did individually search our endoscopy data for cirrhosis and confirmed with clinical, laboratory, radiologic, or pathology data supporting a cirrhosis diagnosis. Although we have a large population of patients with cirrhosis with a robust number of surveillance endoscopies, our numbers of patients with cirrhosis and $\mathrm{BE}$ are small which may have limited our ability to assess statistical differences between patient population. Additionally given the large volume of short segment $\mathrm{BE}$, there may have been a number of false positives leading to an over estimate of SBE.

\section{Conclusion}

In conclusion, a higher prevalence of Barrett's esophagus is noted within our population of cirrhotic patients undergoing endoscopy. Patients with cirrhosis often undergo endoscopy for cirrhosis related issues such as screening for varices or evaluation of gastrointestinal bleeding and surveillance of Barrett's esophagus may be missed. As patients with cirrhosis and Barrett's esophagus tend to have poorer outcomes and tolerate therapies for esophageal cancer less well, it is important to recognize possible shared risk factors and the increased prevalence of Barrett's esophagus and continue endoscopic surveillance in this patient population. ${ }^{21}$

\section{Acknowledgments}

None.

\section{Conflict of interest}

Author declares that there is no conflict of interest.

\section{References}

1. Kamangar F, Fores GM, Anderson WF. Patters of cancer incidence, mortality, and prevalence across five continents: defining priorities to reduce cancer disparities in different geographic regions of the world. $J$ Clin Oncol. 2006;24(14):2137-2150.

2. Pohl H, Welch HG. The role of overdiagnosis and reclassification in the marked increase of esophageal adenocarcinoma incidence. J Natl Cancer Inst. 2005;97(2):142-146.
3. Trivers KF, Sabatino SA, Stewart SL. Trends in esophageal cancer incidence by histology, United States, 1998-2003. Int J Cancer. 2008;123(6):1422-1428.

4. Alcedo J, Ferrández A, Arenas J, et al. Trends in Barrett's esophagus diagnosis in Southern Europe: implications for surveillance. Dis Esophagus. 2009;22(3):239-248.

5. Singh S, Sharma AN, Murad MH Buttar NS, et al. Central adiposity is associated with increased risk of esophageal adenoma, metaplasia, and adenocarcinoma: a systemic review and meta-analysis. Clin Gastroenterol Hepatol. 2013;11(11):1399-1412.

6. Rubenstein JH, Mattek N, Eisen G. Age and sex specific yield of Barrett's esophagus by endoscoy indication. Gastrointest Endosc. 2010;71(1):21-27.

7. Rubenstein JH, Morgenstern H, Appelman H, et al. Prediction of Barrett's esophagus among men. Am J Gastroenterol. 2013;108(3):353-362.

8. Sorensen HT, Friis S, Olsen JH, et al. Risk of liver and other types of cancer in patients with cirrhosis: a nationwide cohort study in Denmark. Hepatology. 1998;28(4):921-925.

9. Randi G, Altieri A, Gallus S, et al. History of cirrhosis and risk of digestive tract neoplasms. Ann Oncol. 2005;16(9):1551-1555.

10. Kalaitzakis E, Gunnarsdottir SA, Josefsson A, et al. Increased risk for malignant neoplasms among patients with cirrhosis. Clin Gastroenterol Hepatol. 2011;9(2):168-174.

11. Trivin F, Boucher E, Vauléon E, et al. Management of esophageal carcinoma associated with cirrhosis: a retrospective case-control analysis. J Oncol. 2009;2009:173421.

12. Raftopoulos SC, Efthymiou M, May G, et al. Dysplastic Barrett's esophagus in cirrhosis: a treatment dilemma. Am J Gastroenterol. 2011;106(9):1724-1726.

13. Dufour DR, Lott JA, Nolte FS, et al. Diagnosis and monitoring of hepatic injury. II. Recommendations for use of laboratory tests in screening, diagnosis, and monitoring. Clin Chem. 2000;46:2050-2068.

14. Skelly MM, James PD, Ryder SD. Findings on liver biopsy to investigate abnormal liver function tests in the absence of diagnostic serology. $J$ Hepatol. 2001;35(2):195-199.

15. Passaretti S, Mazzotti G, de Granchis R, et al. Esophageal motility in cirrhotics with and without esophageal varices. Scan J Gastroenterol. 1989;24(3):334-338.

16. Bhatia SJ, Narawane NM, Shalia KK, et al. Effect of tense ascites on esophageal body motility and lower esophageal sphincter pressure. Indian J Gastroenterol. 1999;18(2):63-65.

17. Shaheen N, Falk GW, Iyer PG, et al. ACG Clinical Guidelines: Diagnosis and Management of Barrett's Esophagus. Am J Gastroenterol. 2015;111(1):30-50.

18. Richter JE. Role of the gastric refluxate in gastroesophageal reflux disease: acid, weak acid and bile. Am J Med Sci. 2009;338(2):89-95.

19. Garcia-Tsao G, Sanyal AJ, Grace ND, et al. Prevention and management of gastroesophageal varices and variceal hemorrhage in cirrhosis. Am J Gastroenterol. 2007;102(9):2086-2102.

20. Evans JA, Early DS, Fukami N, et al. The role of endoscopy in the management of Barrett's esophagus and other premalignant conditions of the esophagus. Gastrointest Endosc. 2012;76(6):1087-1094.

21. Trivin F, Boucher E, Vauleon E, et al. Management of esophageal carcinoma associated with cirrhosis: A retrospective case-control analysis. J Oncol. 2009;2009:173421. 